\title{
A census of AGB stars in the Milky Way and M31 subgroups of dwarf-spheroidal galaxies
}

\author{
F. Kerschbaum, B. Heiling, W. Nowotny, Ch. Spindler \\ Institut für Astronomie der Universität Wien, Türkenschanzstraße 17, \\ A-1180 Wien, Austria
}

H. Olofsson

SCFAB, Stockholm Observatory / Department of Astronomy, SE-106 91 Stocḱholm, Sweden

H.E. Schwarz

Cerro Tololo Inter-American Observatory, NOAO-AURA, Casilla 603, La Serena, Chile

\begin{abstract}
From photometric observations out to the tidal radii of the galaxies, we were able to identify hundreds of new carbon stars, to derive mean absolute magnitudes $\left\langle M_{i}\right\rangle$, luminosity functions, and the spatial/radial distributions of the carbon stars in these galaxies. For each galaxy this new material will allow estimates of the tips of their RGBs to derive distance moduli, to derive $\mathrm{C} / \mathrm{M}$ ratios of the AGB populations and bolometric magnitudes $M_{b o l}$. At the end of our survey a comparison of all quantities of the late-type stars as a function of the properties of the host galaxies will be made possible.
\end{abstract}

\section{Introduction}

Asymptotic Giant Branch (AGB) stars are interesting objects of study because of their role in stellar evolution and their mass loss contributing significantly to the enrichment of the interstellar medium. This makes them important constituents and probes of extragalactic systems. On the other hand extragalactic studies are important for our understanding of AGB evolution itself. As members of well defined environments or populations, many astrophysical questions are much easier to answer in external galaxies than in our own Galaxy (see motivation in Nowotny et al. 2001).

Therefore the efficient identification and characterization of AGB stars in local group galaxies is of special importance and is now carried out by a number of groups (recently Nowotny et al. 2003; Demers et al. 2003). The photometric method for the identification of AGB stars in crowded fields consists in combining a wide-band temperature criterion (e.g. $V-i$ ) and a narrow-band, chemistrysensitive colour index as described by Palmer \& Wing (1982). 

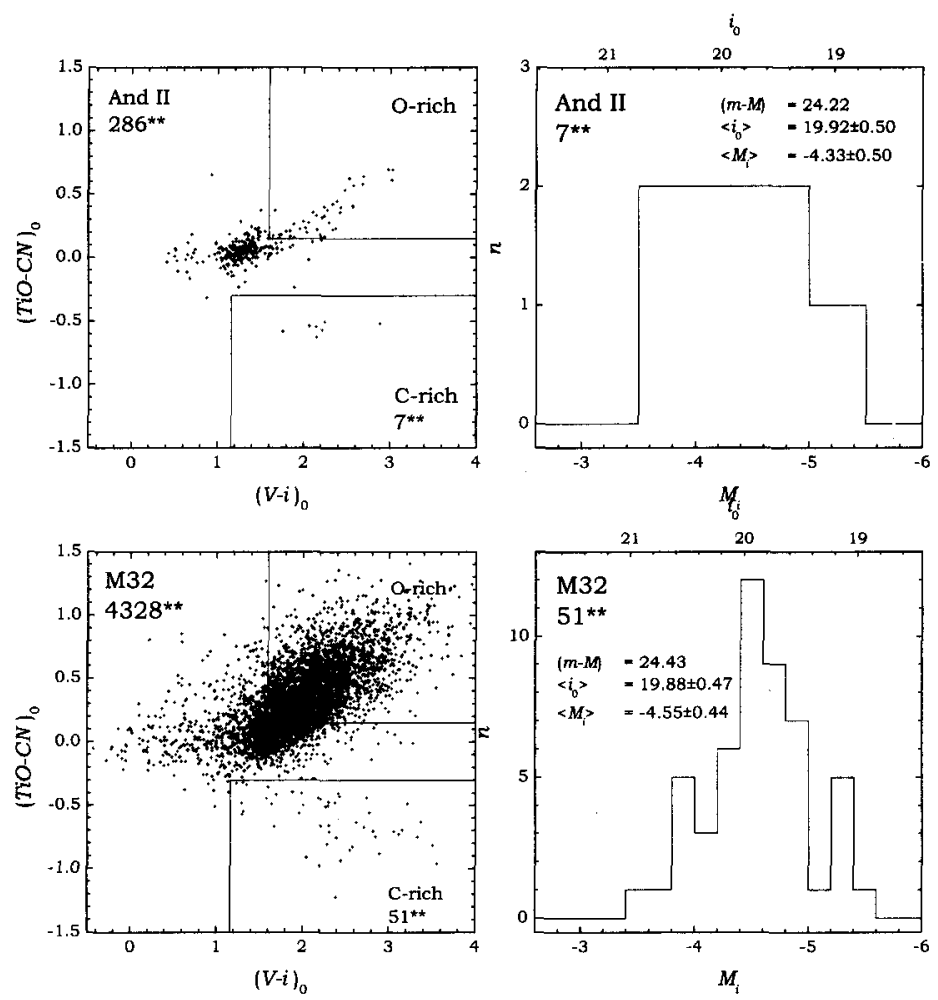

Figure 1. Colour-colour diagrams and $i$-luminosity functions.

\section{New observations}

Following basically the same observational approach as presented in Nowotny et al. (2003) for NCC 147 and NGC 185, four additional galaxies from our local group survey are presented here. Basic data of the galaxies were taken from Mateo (1998) and van den Berg (2000).

\subsection{And II}

In the upper two panels of Fig. 1, a colour-colour diagram and the carbon star (C-star) luminosity function of the faint $\left(M_{V}=-11.22\right)$ dwarf spheroidal And II is shown. Among the 2307 stars probed in the sparse field, seven new C-star candidates with a mean $\left\langle M_{i}\right\rangle=-4.33$ could be identified. Since their spatial distribution is identical with that of the O-rich population, their membership in the And II system is very probable.

\subsection{M32}

The E2 Galaxy M32 at approximately M31's distance of $(m-M)_{0}=24.40$ is especially interesting since it is a matter of debate if it contains an intermediate population at all. Also its tidal interaction with nearby M31 is of interest. As 


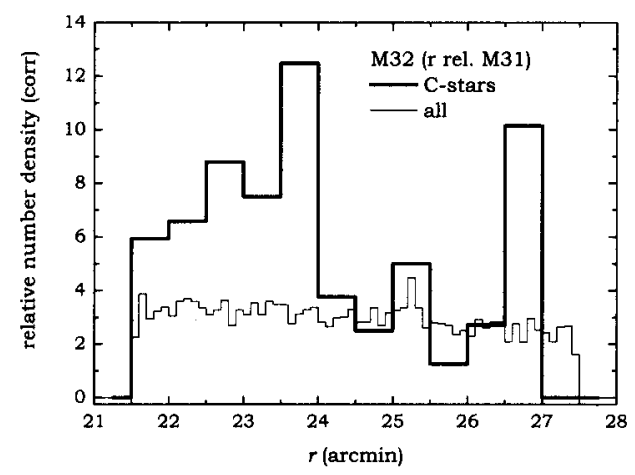

Figure 2. Radial distributions of stars relative to the centre of M31.

seen in the lower two panels of Fig. 1 we find C-stars in the "field" of M32. Both the distribution in the colour-colour diagram as well as the luminosity function of the 51 C-star candidates resemble those of M31 (compare e.g. Nowotny 2001). The radial distributions of stars in the M32 field relative to the centre of M31 presented in Fig. 2 clearly shows that they are not concentrated toward the M32 centre but toward M31. From an extrapolation of the density of C-stars in the disk of M31 (Battinelli et al. 2003) to the M32 position at least two-thirds of them can be attributed to M31 and not M32. The recent announcement of a tidally disrupted galaxy, AndVIII, in the M31/M32 area (Morrison et al. 2003) also could add some interesting aspects to that question.

\subsection{Leo I and Leo II}

Leo I, the low-metallicity dwarf spheroidal at $(m-M)_{0}=22.20$, produced $81 \mathrm{C}$ star candidates in its colour-colour plot leading to a typical mean $\left\langle M_{i}\right\rangle=-4.43$ in the luminosity function that also extends to unusually low values. A detailed comparison with older spectroscopic surveys, infrared work (Menzies at al. 2002) and a similar study by Demers \& Battinelli (2003) will be carried out soon. As shown in Fig. 3, Leo II, somewhat closer at $(m-M)_{0}=21.60$, and fainter, $M_{V}=-10.07$, allows the identification of 15 C-star candidates with a very low mean absolute $\left\langle M_{i}\right\rangle=-3.7$. As in the case of Leo I, a detailed comparison (including object by object) with older work, especially spectroscopic studies, is needed to clarify the nature of these low luminosity red giant stars!

\section{Summary and outlook}

To date we have published our results on M31, NGC 147 and NGC 185. For And II, M32, Leo I and Leo II, data reduction and preliminary analysis has been done. For the Draco, UMi, Antlia and DDO 210 dwarf spheroidal galaxies, all observations have been made. At this point the completion of the census is of highest priority. Special emphasis will be put on a comparison of our results with those of similar projects (e.g. Battinelli/Demers group) in order to shed light 

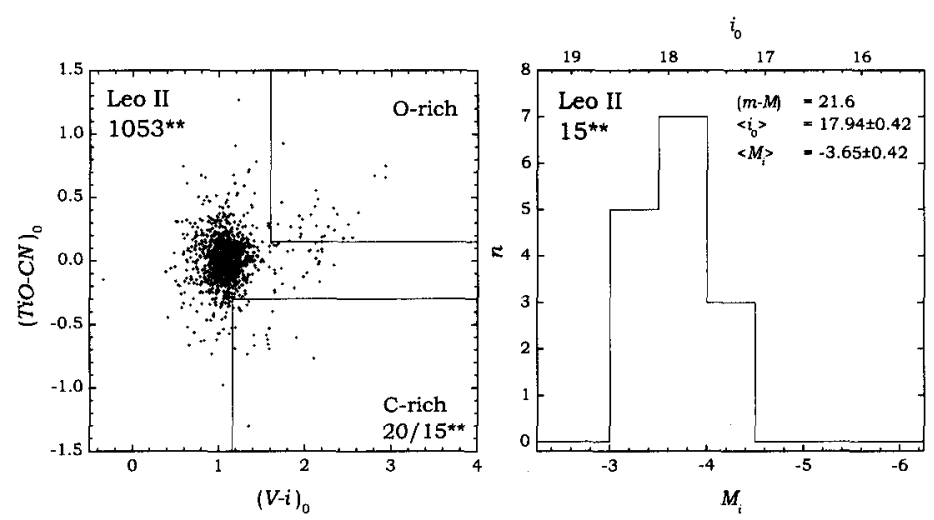

Figure 3. Colour-colour diagram and $i$-luminosity function for Leo II.

on the effects of the different selection of filters (slightly different narrow-band systems and the use of Johnson $R$ instead of $V$ as selected by us).

The knowledge we gained about the chemistry of the singled-out AGB stars can be supplemented by variability information (monitoring of selected galaxies is just starting). Supplemental near-infrared imaging can deliver both bolometric corrections as well as mass-loss information. In the more distant cases where no 2MASS data are available directly, near-infrared imaging is foreseen. Follow-up spectroscopy of selected targets can deliver more detailed information about the stellar properties (e.g. emission lines, effective temperatures, elemental abundances) as well as dynamical information on their host galaxies.

Acknowledgments. This work was supported by the Fonds zur Förderung der Wissenschaftlichen Forschung under the project numbers S7308-AST and P14365-PHY and the Austrian Federal Ministry of Transport, Innovation and Technology in the course of the Project FIRST-PACS. We used the Simbad database operated at CDS, Strasbourg, France.

\section{References}

Battinelli, P., Demers, S., Letarte, B. 2003, AJ, 125, 1298

Demers, S., Battinelli, P. 2002, AJ, 123, 238

Demers, S., Battinelli, P., Letarte, B. 2003, AJ, 125, 3037

Mateo, M. 1998, ARA\&A, 36, 435

Menzies, J., Feast, M., Tanabe, T., Whitelock, P., Nakada, Y. 2002, MNRAS, 335,923

Morrison, H.L., Harding, P., Hurley-Keller, D., Jacoby, G. 2003, ApJL, 596, L183

Nowotny, W., Kerschbaum, F., Olofsson, H., Schwarz, H.E. 2003, A\&A, 403, 93

Nowotny, W., Kerschbaum, F., Schwarz, H.E., Olofsson, H. 2001, A\&A, 367, 557 Palmer, L.G., Wing, R.F. 1982, AJ, 87, 1739

van den Bergh, S. 2000, in The Galaxies of the Local Group, (Cambridge University Press, Cambridge) 


\section{Discussion}

Feast: Is the faint limit for the C stars in Leo I set by your observational limit or is it intrinsic?

Kerschbaum: The limit seems intrinsic. We would detect them at least two mags fainter. One also finds such low luminosity C-stars in the SMC.

Habing: Could the interstellar matter in NGC 185 be a high-velocity cloud captured by that galaxy?

Kerschbaum: NGC 185 shows clear indication of gas/dust plus a young stellar component.

Alves: The idea (from Alves et al. 1998) that all of the red variables may be TP-AGB stars is based on the coincidence of the onset of pulsation with the lowest luminosity of thermal pulsing AGB stars.

Kerschbaum: Our sample goes $\sim 0.5-0.7 \mathrm{mag}$ below the lowest luminosity of thermally pulsing AGB stars.

Alves: Why do Hipparcos stars on the RGB show a dramatic edge in terms of the fraction of variables out of all stars (i.e. $0-100 \%$ ) while in the LMC there is an intermediate range (about 1 mag below TRGB) where about $50 \%$ of stars are variable?

Kerschbaum: In my opinion, there are different biases in those datasets, which make the direct comparison of variability fractions difficult. The amplitudes below the TRGB decreased with decreasing luminosities, but that does not mean necessarily that variability really disappears.

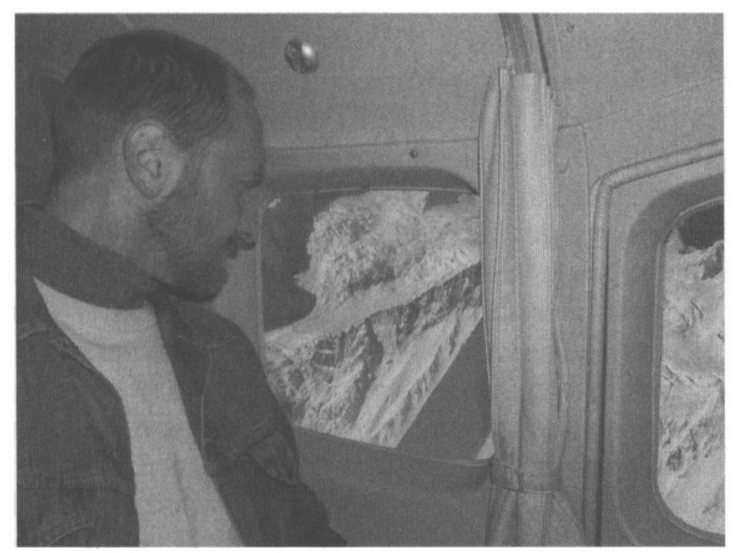

Franz Kerschbaum on the scenic flight over Mt Cook 